\title{
Isolated anterior urethral recurrence of prostatic adenocarcinoma
}

\author{
Darren Beiko, MD, MBA, FRCSC; Khaled Zaza, MD, FRCPC; Kevin V. Power, MD, FRCSC;* \\ D. Robert Siemens, MD, FRCSC;, Alexander H. Boag, MD, FRCPC ${ }^{\S}$
}

`Department of Urology, Queen's University, Kingston General Hospital, Kingston, ON; 'Division of Radiation Oncology, Queen's University, Kingston General Hospital, Kingston, ON; §Department of Pathology, Queen's University, Kingston General Hospital, Kingston, ON; *Division of Urology, Department of Surgery, Queensway Carleton Hospital, Ottawa, ON

Cite as: Can Urol Assoc J 2014;8(5-6):e361-3. http://dx.doi.org/10.5489/cuaj.1692 Published online May 21, 2014.

\section{Abstract}

Isolated anterior urethral metastases from prostate cancer are rare. The pathogenesis of this form of loco-regional recurrence may be related to spread by instrumentation-induced implantation and could potentially then be associated with a better prognosis than metastatic disease secondary to a more malignant phenotype. We report a case of a 68-year-old man with high-risk prostate cancer, diagnosed at transurethral resection of prostate, who was originally treated with combination external beam radiotherapy and hormonal therapy. He re-presented 4 years after his original diagnosis with recurrent gross hematuria and cystourethroscopic biopsies of anterior urethral polyps revealing isolated recurrent prostatic adenocarcinoma. We present our this case and review the literature.

\section{Introduction}

Isolated anterior urethral recurrence of prostate cancer is rare, with about 10 cases reported to date..$^{1-10}$ The precise mechanism of spread is not fully understood, but it is likely related to implantation from previous instrumentation. ${ }^{1,8} \mathrm{We}$ report an interesting case of recurrence of prostatic adenocarcinoma in the bulbous and pendulous urethra in a patient presenting with gross hematuria.

\section{Case report}

A 68-year-old male presented with a 2-week history of gross hematuria with passage of large blood clots per urethra that were causing obstructive lower urinary tract symptoms. His medical history was significant for recent deep venous thrombosis with pulmonary emboli, first-degree atrioventricular block, paroxysmal atrial fibrillation, gout and, impor- tantly, prostate cancer. At the time of presentation, he was on anti-coagulation therapy with warfarin.

Four years earlier, the patient was diagnosed with highrisk prostate cancer following a transurethral resection of the prostate (TURP) performed for urinary retention and resultant acute renal failure. The TURP revealed that $30 \%$ of the specimen contained Gleason $4+5=9 / 10$ prostatic adenocarcinoma, with clear cell or hypernephroid morphology. Further staging demonstrated Stage III disease, Gleason grade 9/10, serum prostate-specific antigen (PSA) of $3.31 \mu \mathrm{g} / \mathrm{L}$ and no evidence of distant metastases on computed tomography (CT) scan of the abdomen and pelvis or bone scan. He elected to pursue combination therapy with external beam radiation therapy and androgen deprivation therapy (ADT) for 2 years receiving 2 Gy fractions over 7 weeks for a total of 70 Gy. Two years after the original diagnosis, he had a PSA nadir of $0.17 \mu \mathrm{g} / \mathrm{L}$.

When he presented 4 years after the original diagnosis, his PSA was stable at $0.7 \mu \mathrm{g} / \mathrm{L}$ and imaging was unremarkable. Cystourethroscopy revealed a single 2-mm polyp in the midbulbous urethra posterolaterally at the $10 \mathrm{o}^{\prime}$ clock position on the right side. Cold cup excisional biopsy was performed and fulguration was unnecessary because of negligible bleeding and complete removal of the polyp. Histopathology revealed a complex proliferation of atypical glandular cells showing confluent zones of cribriform growth consistent with prostatic adenocarcinoma that was morphologically similar to the tumour in the original TURP (Fig. 1). The diagnosis was supported by immunohistochemistry (IHC) stains that showed the abnormal cells positive for racemase, prostatic acid phosphatase and PSA (Fig. 2). IHC for basal cell markers (high molecular weight keratin and p63) showed only focal staining around the abnormal glands, which likely represented an entrapped urethral epithelium.

He re-presented 3 months later with recurrent gross hematuria and cystourethroscopy, again revealing 4 tiny $(<2 \mathrm{~mm}$ each) circumferential polypoid lesions in the bulbous and pendulous urethra. Biopsy and complete fulguration were 


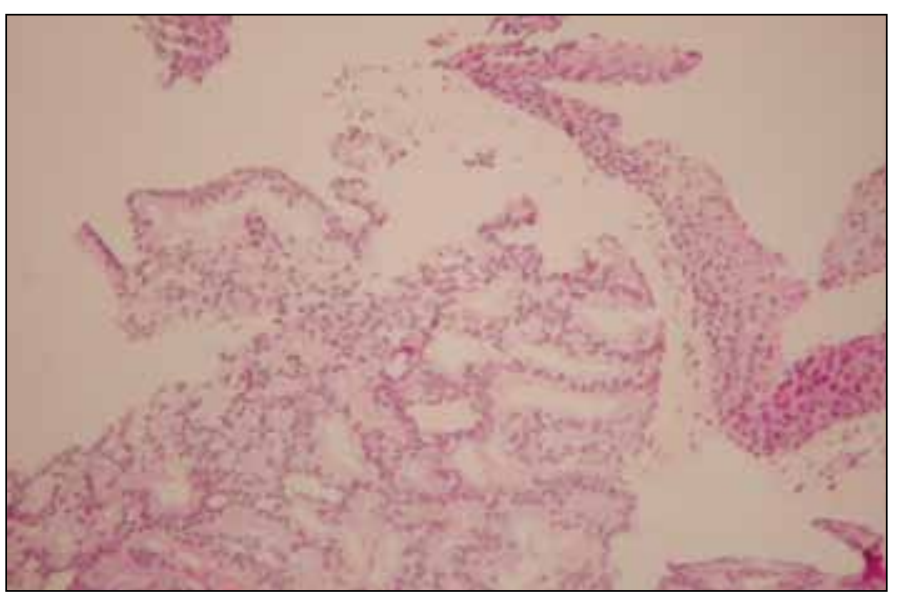

Fig. 1. Histology of the bulbous urethral biopsy showed a cribriform pattern of prostatic adenocarcinoma.

carried out, with no need for a formal TUR. Pathology confirmed recurrent metastatic prostatic adenocarcinoma. Given the clinical scenario of local recurrence with a low PSA and negative imaging, the patient decided on watchful waiting to avoid further ADT.

Over the next 2 years, his PSA slowly rose to $13.4 \mu \mathrm{g} / \mathrm{L}$ and he was re-started on ADT with a PSA nadir of only $2.7 \mu \mathrm{g} / \mathrm{L}$. Over the next 2 years following re-initiation of ADT, his PSA continued to rise to $10.4 \mu \mathrm{g} / \mathrm{L}$, and he required a repeat TURP for bleeding and progressive lower urinary tract symptoms. He was managed for his M0, castration resistant prostate cancer (CRPC), with a relatively slow doubling time. During this time he was concurrently managed for his metastatic small cell lung cancer. A multidisciplinary discussion of the case confirmed that the patient's lung disease was most likely not secondary to his prostate cancer diagnosis given the clinical, imaging and pathological findings. The patient subsequently died from his small cell carcinoma of the lung, 4 years following the diagnosis of his anterior urethral prostate cancer recurrence.

\section{Discussion}

Although the mechanisms of invasion, migration and metastases are multiple and complex, loco-regional spread of prostate cancer has been demonstrated by direct tumour penetration, perineural space invasion, ${ }^{11}$ lymphatic invasion, ${ }^{1}$ vascular invasion ${ }^{12}$ and extension through the ejaculatory ducts. ${ }^{13}$ Metastasis of prostatic adenocarcinoma to the anterior urethra is rare and its pathogenesis is misunderstood. Several theories for the metastatic spread of prostatic adenocarcinoma to the anterior urethra have been proposed, including implantation following instrumentation, ${ }^{2,8}$ direct extension, arterial tumour emboli, retrograde venous dissemination and retrograde lymphatic spread. ${ }^{1,8,10}$ Most of the previously reported cases with prostate cancer and anterior

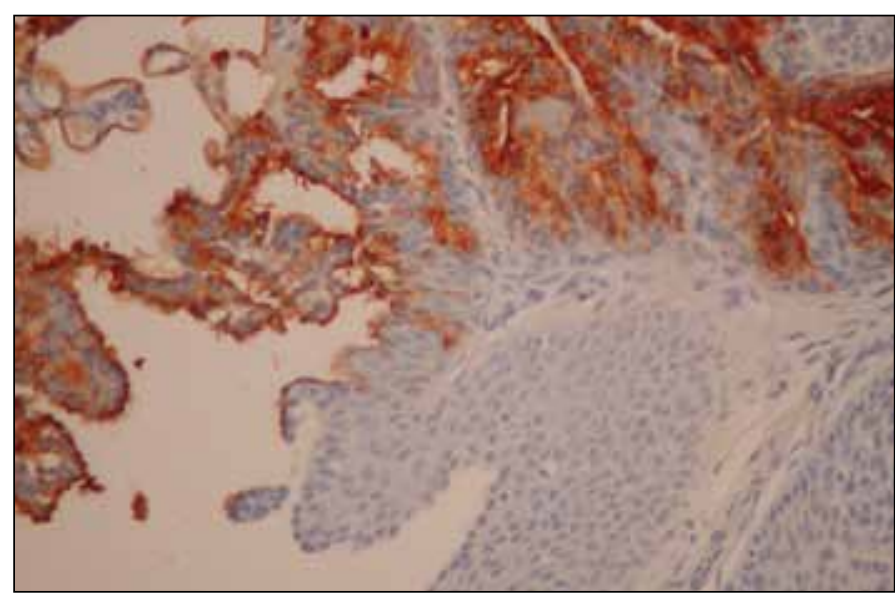

Fig. 2. Immunohistochemical stain of the bulbous urethral biopsy was positive for prostate-specific antigen.

urethral metastasis had prior instrumentation with TURP or urethral catheterization. ${ }^{1-10}$ As implantation of cancer deposits by instrumentation may not be representative of the malignant process required for distant metastases, it has been suggested that this clinical scenario may portend a more favorable prognosis. ${ }^{1}$

There are no specific recommendations regarding treatment of isolated anterior urethral metastasis from prostatic adenocarcinoma. Reported treatment options include TUR, fulguration or ablation of tumour, ${ }^{2,5}$ systemic ADT, ${ }^{4}$ topical chemotherapy, radiotherapy and more radical surgery, including urethrectomy. ${ }^{8}$ We had hypothesized that the most likely mechanism of recurrence in this reported case was post-TURP implantation. The sparse literature at the time of presentation and the low PSA values led us to consider conservative management, specifically given his concurrent diagnosis of lung cancer.

\section{Conclusion}

This is an uncommon case of recurrent anterior urethral metastasis after management of prostate cancer. The natural history in this case of high-risk disease, including local recurrence and the development CRPC within 6 years of diagnosis, was nonetheless relatively indolent with no documented visceral or bony metastases. This case supports the literature suggesting that recurrence of prostate cancer in the anterior urethra is not necessarily a poor prognostic marker; it is consistent with biology allowing local deposition and growth, due to instrumentation, without an associated aggressive metastatic phenotype.

Competing interests: Dr. Beiko, Dr. Zaza, Dr. Power and Dr. Boag all declare no competing financial or personal interests. Dr. Siemens is Editor-in-Chief at CUAJ. 
This paper has been peer-reviewed.

\section{References}

1. Taylor GB, MCNeal JE, Cohen RJ. Intraductal carcinoma of the prostate metastatic to the penile urethra: A rare demonstration of two morphologic patterns of tumor growth. Pathology 1998;30:218-21. http:// dx.doi.org/10.1080/00313029800169306

2. Blanco Díez A, Ruibal Moldes M, Rodríguez-Rivera García J, et al. Metastasis of prostatic carcinoma to the urethra: Report of a case [in Spanish]. Actas Urol Esp 2003;27:735-8. http://dx.doi.org/10.1016/ S0210-4806(03)73006-X

3. Subudhi CL, Panda SN, Panigrahi KK. Urethral metastases from carcinoma of prostate. Indian J Cancer 1994:31:31-3.

4. Koh E, Sekii K, Namiki $M$, et al. Urethral metastasis from prostatic carcinoma as diagnosed by immunoperoxidase technique using prostate-specific antigen and prostate-specific acid phosphatase. Eur Urol 1987;13:142-4.

5. Rao MS, Bapna $B C$, Bhat VN, et al. Multiple urethral metastases from prostatic carcinoma causing urinary retention. Urology 1977;10:566-7. http://dx.doi.org/10.1016/0090-4295(77)90104-2

6. Iverson AP, Blackard CE, Schulberg VA. Carcinoma of the prostate with urethral metastases. I Urol 1972;108:901-4.
7. Kotecha N, Gentile RL. Carcinoma of prostate with urethral metastasis. Urology 1974;3:85-6. http:// dx.doi.org/10.1016/50090-4295(74)80070-1

8. Green JM, Tang WW, Jensen BW, et al. Isolated recurrence of ductal prostate cancer to anterior urethra. Urology 2006;68:428. http://dx.doi.org/10.1016/i.urology.2006.03.024

9. Nabi G, Devinder S, Dogra RN, et al. Malignant anterior urethral strictures: A rare complication of transurethral resection of malignant prostate. Int Urol Nephrol 2002;34:73-4. http://dx.doi. org/10.1023/A:1021364224604

10. Kobayashi T, Fukuzawa $\mathrm{S}, \mathrm{Oka} \mathrm{H}$, et al. Isolated recurrence of prostatic adenocarcinoma to the anterior urethra after radical prostatectomy. J Urol 2000;164:780. http://dx.doi.org/10.1016/S0022$5347(05) 67306-5$

11. Villers A, McNeal JE, Redwine EA, et al. The role of perineural space invasion in the local spread of prostatic adenocarcinoma. J Urol 1989;142:763-8.

12. Zhang $S$, Wang $X$, Osunkoya $A 0$, et al. EPLIN downregulation promotes epithelial-mesenchymal transition in prostate cancer cells and correlates with clinical lymph node metastasis. Oncogene 2011;30:4941-52. http://dx.doi.org/10.1038/onc.2011.199

13. Mirowitz SA. Seminal vesicles: Biopsy-related hemorrhage simulating tumor invasion at endorectal MR imaging. Radiology 1992;185:373-6.

Correspondence: Dr. Darren Beiko, Associate Professor, Department of Urology, Queen's University, Kingston General Hospital, 76 Stuart St., Kingston, ON K7L 2V7; beikod@kgh.kari.net 